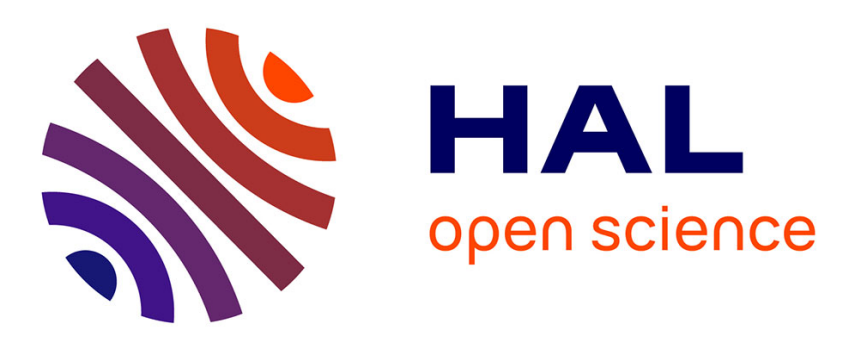

\title{
Sumatra Nord-Est dans l'espace acihais jusqu'à la fin de la guerre d'Aceh
}

\author{
Daniel Perret
}

\section{To cite this version:}

Daniel Perret. Sumatra Nord-Est dans l'espace acihais jusqu'à la fin de la guerre d'Aceh. Archipel, 1994, 48 (1), pp.63-86. 10.3406/arch.1994.3002 . halshs-01877458

\section{HAL Id: halshs-01877458 \\ https://shs.hal.science/halshs-01877458}

Submitted on 19 Sep 2018

HAL is a multi-disciplinary open access archive for the deposit and dissemination of scientific research documents, whether they are published or not. The documents may come from teaching and research institutions in France or abroad, or from public or private research centers.
L'archive ouverte pluridisciplinaire HAL, est destinée au dépôt et à la diffusion de documents scientifiques de niveau recherche, publiés ou non, émanant des établissements d'enseignement et de recherche français ou étrangers, des laboratoires publics ou privés. 


\section{Sumatra Nord-Est dans l'espace acihais jusqu'à la fin de la guerre} d'Aceh

\section{Daniel Perret}

\section{Citer ce document / Cite this document :}

Perret Daniel. Sumatra Nord-Est dans l'espace acihais jusqu'à la fin de la guerre d'Aceh. In: Archipel, volume 48, 1994. pp. 6386 ;

doi : https://doi.org/10.3406/arch.1994.3002

https://www.persee.fr/doc/arch_0044-8613_1994_num_48_1_3002

Fichier pdf généré le 21/04/2018 
ETUDES

Daniel PERRET

\section{Sumatra nord-est dans l'espace acihais jusqu'à la fin de la guerre d'Aceh}

Depuis sa fondation, le sultanat d'Aceh s'efforce de contrôler les richesses situées hors de son territoire immédiat. Cette situation suppose l'existence d'une organisation coercitive, ou librement acceptée, capable d'assurer une régularité dans l'approvisionnement de manière à fidéliser les marchands étrangers. Parmi les régions pourvoyeuses, nous examinerons ici le cas de Sumatra nord-est, et en particulier celui des sultanats de Deli et Serdang (et de leur arrière-pays limitrophe du lac Toba, les «pays Karo» et «Simalungun»). Nous avons montré ailleurs que l'interdépendance des populations côtières et intérieures permet de considérer l'espace ainsi défini comme un espace cohérent (1). A l'avantage économique tiré du contrôle d'une région riche en ressources naturelles et agricoles comme peut l'être Sumatra nord-est, s'ajoutent d'autres potentialités susceptibles d'intéresser Aceh: la possibilité de faire de cette région une base avancée pour attaquer la ville de Malaka alors aux mains des Portugais, et de répandre l'islam parmi une population encore majoritairement païenne.

La grande difficulté pour étudier l'histoire des liens unissant Sumatra nord-est et Aceh réside dans le caractère fragmentaire des sources à notre disposition. Les informations sont rares jusqu'au début du XIXe siècle. En effet, les dirigeants côtiers ont toujours empêché les marchands étrangers de pénétrer dans l'arrièrepays où se trouvaient l'essentiel des ressources commercialisables. C'est pourquoi les témoins occidentaux du XVIe siècle et du XVIIe siècle ignorent-ils tout en ce domaine et même Marsden, à la fin du XVIIIe siècle, reste très évasif, indiquant seulement que le royaume d'Aceh ne s'étend pas «au-delà de quarante à cinquante miles dans l'intérieur». Snouck Hurgronje, quant à lui, affirme que durant sa courte période de prospérité, le sultanat n'a jamais exercé de contrôle effectif sur l'arrièrepays ${ }^{(2)}$. Les sources acihaises, déjà très peu prolixes sur l'administration de l'immédiat arrière-pays, le sont a fortiori encore moins pour ce qui concerne les régions périphériques (3). 
Pourtant l'histoire de Sumatra nord-est recèle nombre d'indices permettant d'avancer l'idée que cette région s'est trouvée plus ou moins intégrée dans la sphère acihaise probablement tout au long de l'existence du sultanat. La fragilité de la chronologie, liée à la trop grande fragmentation des sources, ne nous permet pas de cerner des phases bien délimitées d'intensité ou d'affaiblissement de ces relations. C'est pourquoi nous avons choisi de présenter ces relations par thèmes: l'économique, le politique, le religieux et le culturel. Ce découpage est bien entendu artificiel et nous essaierons, de mettre en rapport ces différentes sphères dans la conclusion.

\section{Sumatra nord-est dans l'espace économique acihais}

\section{Ressources naturelles et chevaux}

Très tôt le nord de Sumatra est connu comme une région riche en produits forestiers. A côté de différentes résines ${ }^{(4)}$ et gommes ${ }^{(5)}$, il pouvait également fournir de la cire, des défenses d'éléphants (6), de l'or (7) et du pétrole ${ }^{(8)}$. Dans sa description d'Aru (9) vers 1515, Tomé Pires signale que les produits forestiers que commercialise ce royaume sont, pour partie, envoyés à Pasai et Pedir (10). Il est logique de penser qu'une décennie plus tard, au moment où Aceh commence à exercer sa suprématie sur l'extrême nord de l'île, les produits d'Aru sont alors convoyés vers Aceh. Si les sources sont muettes sur les modalités d'acheminement vers Aceh de ces produits naturels, il est fort probable que les arrivages de produits d'Aru s'intensifient à partir de la deuxième moitié du XVIe siècle, notamment entre 1564 et 1589 , au moment où Aru est sous l'autorité du sultanat.

A côté des produits forestiers et minéraux, le cheval a pu faire partie des ressources locales intéressant Aceh. Nous savons en effet qu'au moins dès le XVIIe siècle, il est utilisé dans l'armée du sultanat (11). Or, les sources hollandaises de l'époque mentionnent cet animal comme un produit d'exportation de Deli (12), qui a été conquis par Aceh en 1612. Ils sont de suffisamment bonne qualité pour que le général en chef de Deli en envoie un comme présent au gouverneur de Malaka (13). Par ailleurs, le Pustaka Kembaren, un manuscrit pré-colonial du nord du lac Toba, fait état d'un commerce de chevaux entre le plateau situé au nord du lac Toba et Langkat, un territoire voisin d'Aceh (14). Le grand centre d'approvisionnement devait être l'île de Samosir sur le lac Toba, où l'on sait que les bêtes étaient achetées jeunes pour être élevées au nord et à l'est du lac. Ce commerce avec Deli et Langkat était vraisemblablement monopolisé par les chefs du plateau au nord du lac Toba car c'est sur leur territoire qu'étaient situés les passages les plus aisés vers la côte (15). Là encore, si un tel commerce avec Aceh a pu exister, nous n'en connaissons pas l'organisation.

\section{Aceh, stimulateur de l'agriculture locale?}

Nous posons cette question à propos de deux cultures qui, pour des raisons différentes, ont pu être encouragées par le grand sultanat du nord. Il s'agit du riz et du poivre. 
Au cours du XVIIe siècle, la principale richesse agricole du nord-est de Sumatra est le riz. Deli, qui prend le relais d'Aru, semble en effet mettre d'abord l'accent sur un développement de la production rizicole, basé sur une volonté de défrichements qui s'affirme avec l'importation de ferraille (16) nécessaire à la confection d'outils performants. Peu après leur installation à Malaka en 1641, les Hollandais auront d'ailleurs tôt fait de repérer les importantes capacités de production de Deli dans ce domaine (17). Il y a tout lieu de se demander si Aceh n'est pas intervenu pour stimuler l'expansion de cette culture dans la région. Nous savons en effet que tout au long de son histoire le sultanat d'Aceh fut confronté au problc̀mc crucial de l'approvisionnement en riz pour nourrir sa population ainsi que les étrangers venant faire du commerce (18). Or dès le début du XVIe siècle, le plateau situé au nord du lac Toba ainsi que la plaine côtière peuvent faire office de «greniers à riz» (19) et un routier portugais de la fin du XVIe siècle mentionne Aru comme principal fournisseur insulindien du sultanat en ce qui concerne les vivres (20). Une tradition de l'ouest du plateau au nord du lac Toba pourrait constituer un autre indice de cette relation. En effet, à la fin du XIXe siècle, on croit encore à Kuala que le riz ne réussit qu'aussi longtemps que les habitants accordent le passage aux Acihais (21). Nous savons également que, lors de la guerre qui oppose Néerlandais et Acihais à partir de 1873 jusque dans les premières années du XXe siècle, le blocus côtier imposé par les autorités coloniales entraîne l'intensification des incursions sur le plateau au nord du lac Toba de groupes venus du sultanat pour s'approvisionner en riz (22).

Le nord-est de Sumatra a probablement aussi contribué d'une autre manière au développement de la riziculture pour le compte d'Aceh. En effet, l'important prélèvement humain effectué par Aceh après la conquête de Deli, en 1612, visait sans nul doute à recruter principalement des agriculteurs pour cultiver le riz aux abords de la capitale (23). Cette déportation massive constitue peut-être l'inauguration par le sultan Iskandar Muda de ce qui deviendra une «tradition» encore vivante au milieu du XIXe siècle, à savoir la présence d'esclaves originaires de Sumatra nord-est sur le territoire même du sultanat (24).

A côté du développement de la riziculture, se pose la question du rôle d'Aceh dans l'expansion des plantations de poivriers à Sumatra nord-est. Si, en ce qui concerne la moitié nord de l'île, Pasai se manifeste en premier dans les sources (au début du XVe siècle) comme producteur de poivre ${ }^{(25)}$, nous ne disposons pas d'éléments permettant de dater avec précision l'apparition de ces plantations sur la côte nord-est de Sumatra (26). Alors que, dans la première moitié du XVIe siècle, ni Pires ni Pinto ne citent le poivre comme une production de cette région, le routier de Dom Joao Ribeiro Gaio, daté d'environ 1588, indique que le poivre vendu à Aceh vient aussi d'Aru (27).

Pourtant au siècle suivant, aucun des daghregister publiés (1624-1682) ne fait allusion à la culture du poivrier sur la côte est de Sumatra-Nord. Même s'il est connu que les récoltes de poivre sont très irrégulières, si le poivrier ćtait dćjà cultivé dans la région à la fin du XVIe siècle, il est difficile de s'expliquer cette absence dans les sources durant les quarante premières 
années du gouvernement de Malaka par les Bataves. Les premiers fonctionnaires néerlandais à s'installer dans la région, au cours des années 1860, indiquent simplement que les tribus «batak» du nord de Sumatra ont, depuis de nombreuses années, planté presque exclusivement du poivrier (28).

La première mention certaine remonte au XVIIIe siècle dans un rapport britannique indiquant que Langkat, frontalier avec Aceh, produit du poivre (29). D'autre part, un témoignage recueilli par un fonctionnaire néerlandais révèle qu'à la même époque, deux personnages originaires de Siak, Said Amat et Said Ali, auraient été chassés et auraient émigré vers Langkat où ils se seraient enrichis grâce au commerce du poivre (30). Dans cc sultanat frontalicr avec Aceh, la tradition veut que la culture du poivrier ait été exclusivement une activité exercée par des immigrants originaires de la côte nord et sud d'Aceh (31). D'ailleurs, au début du XXe siècle, de nombreux Acihais cultivent encore des poivriers au nord de Langkat, notamment autour de la baie d'Aru (32). Tel ne semble pas avoir été le cas dans les sultanats de Deli et Serdang, où le premier témoignage direct sur ces plantations émane de John Anderson qui visite la région en 1823 .

Cependant, certains indices portent à croire qu'Aceh a pu jouer un rôle dans le développement de ces cultures à Deli. Parmi quatre personnages autochtones mis en scène dans les généalogies et les traditions locales sinon comme les promoteurs, du moins comme des entrepreneurs dynamiques qui vont développer ces plantations, deux sont dits avoir séjourné temporairement à Aceh.

Le premier, Si Emblang Pinggol, originaire de Barus, va à Aceh où il épouse la fille d'une importante autorité locale avant de revenir à Barus, puis de traverser le plateau au nord du lac Toba. Il fonde Barusjahe dans l'arrièrepays de Deli, puis Pertumbukan dans le piémont du sultanat de Serdang (33). On sait que Pertumbukan est un endroit riche en plantations de poivriers dans la seconde moitié du XIXe siècle (34). Si Emblang Pinggol est l'ancêtre supposé des dirigeants (kejuruan) de la principauté (urung) de Senembah, l'un d'eux qui, selon la généalogie, appartient à la sixième génération (35), est nommé kejuruan en 1862. Ces éléments renvoient par conséquent la fondation de Pertumbukan à la première moitié du XVIIIe siècle.

Le second, Si Mbelang Cuping ( «Grandes Oreilles»), originaire de Osang, au nord de Sidikalang, à l'ouest du lac Toba, traverse le plateau pour fonder Barusjulu, près de l'actuel Barusjahe. Il entre ensuite dans l'arrière-pays de Deli où il fonde Simeme, au sud de Deli Tua. Devenu riche (nous pensons qu'il s'enrichit grâce à la culture du poivre), il se rend à Aceh pour chercher un titre (pangkat) et se marie avec une Acihaise. Revenu à Deli, Si Mbelang Cuping épouse une fille de la région avec qui il a un fils qui deviendra «noble» (sibayak) de Barusjahe et l'ancêtre de plusieurs dirigeants de l'urung de Si Pitu Kuta sur le plateau. Nous savons également que dans les années 1920, le sibayak de Namo Suro appartenait à la neuvième génération des descendants de Si Mbelang Cuping (36), ce qui ferait remonter les activités de ce dernier sur la côte est à la première moitié du XVIIIe siècle, tout comme celles de Si Emblang Pinggol. 


\section{Sumatra nord-est comme marché périphérique}

Si Aceh s'est attaché à prélever dans la région des ressources nécessaires à sa propre expansion, il semble qu'en retour, sa demande ait favorisé le développement d'une classe autochtone suffisamment riche pour pouvoir acquérir des produits plus ou moins luxueux en provenance du sultanat du nord, tout particulièrement des vêtements et des armes. Plusieurs témoignages du milieu du XVIIe siècle révèlent en effet qu'Aceh fournit Deli en vêtements (37). Au début du XVIIIe siècle, la côte nord-est apparaît fermée aux marchands étrangers (38), mais il est fort probable que les arrivages de marchandises en provenance d'Aceh se soient poursuivis. Nous en voulons pour preuve ce témoignage éloquent de Netscher, à l'époque responsable de la côte est, qui se rend à Deli en 1862. Il décrit ainsi l'ambiance dans la maison du sultan: «[...] C'est un spectacle particulier que de voir rassemblés dans la maison du sultan de Deli quelques deux cents individus assis sur des nattes, la plupart en costumes acihais, armés de kriss et de grandes épées acihaises avec des poignées d'argent. [...] Les gens aisés portent des vêtements de type acihais» (39). Il ajoute que certains de ces «kriss» et épées, de modèle acihais, sont fabriqués à Deli (40).

Les premières incursions occidentales dans l'arrière-pays montrent en outre que le marché visé par les articles acihais est bien loin de se cantonner à la côte. Lorsque Van der Tuuk s'aventure en 1852-1853 dans la région au sudouest du lac Toba, il collecte plusieurs termes ou expressions relatifs à des articles de fabrication acihaise ${ }^{(41)}$. De même, la langue parlée au nord du lac Toba conserve encore, dans la première moitié du XXe siècle des termes relatifs à des pièces de vêtements ou des armes blanches de fabrication acihaise. Nous citerons, pour les premières, le padang rusak, le seluwar, le nipes et le uis beka buluh (deux coiffures en soie très coûteuses portées par les personnages importants) ainsi que le tujuh lompat ${ }^{(42)}$; pour les secondes, l'alamang, le kelewang ou galewang, le bawar, le gadebang et le rodos (43). Aceh maintient sa présence économique sur la côte est bien après l'installation des Hollandais. En 1883, Hagen trouve partout sur les grands marchés du plateau au nord du lac Toba, des marchandises et des produits acihais. Ces marchés sont également fréquentés par des commerçants venus du nord ${ }^{(44)}$.

\section{Sumatra nord-est dans l'espace monétaire acihais}

Très tôt Aceh va battre des monnaies, qu'elles soient d'étain ou d'or, et ceci jusqu'au XVIIIe siècle (45). Plusieurs spécimens de ces dernières, appelées mas (46), ont été retrouvées, depuis le début du siècle, sur la côte et dans le piémont de Deli, notamment dans la région de Deli Tua ${ }^{(47)}$.

Sur le plateau au nord du lac Toba, les «savants» traditionnels, qui enseignent la lecture et l'écriture de la langue vernaculaire sont encore, en 1875, rémunérés en monnaie d'or (drahan), très certainement les dirham du nord de l'île (48). A la même époque, les populations de cette région ont encore coutume d'effectuer des offrandes en mas dans les transactions matrimoniales (49). Quant à la région de Simalungun, à l'est du lac Toba, nous savons que la mon- 
naie d'or acihaise était encore en vigueur sur les marchés au début du XXe siècle ${ }^{(50)}$.

\section{Sumatra nord-est dans l'espace politique acihais}

\section{L'emprise côtière}

C'est dans la première moitié du XVIe siècle que tout le nord de Sumatra et la côte nord-est en particulier, commence à subir l'autorité du sultanat d'Aceh. A la fin de 1539 , le roi d'Aceh se présente comme «sultan Alaradim, roi de l'Achem, de Baarros, de Pédir, de Pacém, des domaines de Daia et des Batas, prince de toute la Terre des Deux Mers, méditerranée et océan, des mines de Menancabo et du nouveau royaume d'Aaru [...]» (51).

Nous savons, grâce au témoignage de Mendes Pinto, que ce dernier territoire est conquis cette même année 1539 (52) avant d'être pris, l'année suivante, par Johor et reconquis par Aceh en 1564 (53). Sultan'Ala ad-Din Ri'ajat Syah al-Kahhar confie alors le royaume d'Aru à son fils aîné Sultan Abdullah (54) qui mourra en 1568 à l'occasion d'un siège de Malaka (55). Johor reprend possession d'Aru en 1589 et c'est seulement au début de 1613 que le royaume de la côte tombe à nouveau entre les mains d'Aceh alors gouverné par le sultan Iskandar Muda qui a soumis Deli l'année précédente ${ }^{(56)}$. L'événement est encore fortement ancré dans la mémoire populaire sous plusieurs formes.

La plus connue est la légende de «Poeteri Hidjau», répandue dans l'arrièrepays d'Asahan, à Simalungun, chez les Karo, les Gayo et probablement chez les Alas (57). Dans la version connue à Deli, on raconte que le sultan d'Aceh envoya une délégation afin de demander en mariage Puteri Hijau, fille de Sultan Sulaiman, le dirigeant de Deli Tua. Mais la demande est rejetée. Le sultan décide alors de partir à la tête de son armée attaquer Deli. Après trente jours de combats acharnés, il demande à un de ses subordonnés d'inventer un stratagème susceptible de faire céder l'adversaire. Cette ruse va consister à lancer de l'argent avec un canon dans la direction des ennemis. L'armée de Deli Tua se précipite alors sur la manne, oubliant de défendre la place ${ }^{(58)}$. Alors que l'aîné s'enfuit, le plus jeune frère de Putri Hijau se transforme en canon et inflige de lourdes pertes dans les rangs acihais. Mais le canon surchauffé éclate. L'armée ennemie en profite alors pour envahir la place fortifiée, se saisit de la princesse et la met sur un navire acihais. En abordant le rivage d'Aceh elle est sauvée par son frère transformé en serpent ${ }^{(59)}$.

Le souvenir de ces conflits est également conservé dans certains lieux sacrés (keramat) . Ainsi au bord de la rivière Belumai (Serdang) près de Tanjung, se trouve un keramat panjang (six à sept mètres de long) que les autochtones croient être la tombe d'un chef de guerre (panglima) acihais mort dans un combat contre les troupes de Putri Hijau. De même à Kota Jurung, au bord de la rivière Tuntungan, se trouve le keramat «Tuan Bujur» ou «Tuan Nagor», qui abriterait le corps d'un Acihais tué durant les mêmes affrontements.

D'autres sources locales font état de relations politiques plus paisibles avec lc sultanat du nord. Au premier rang figure la Hikayat Deli, probablement rédigée au début du XIXe siècle, qui rapporte l'installation sur le terri- 


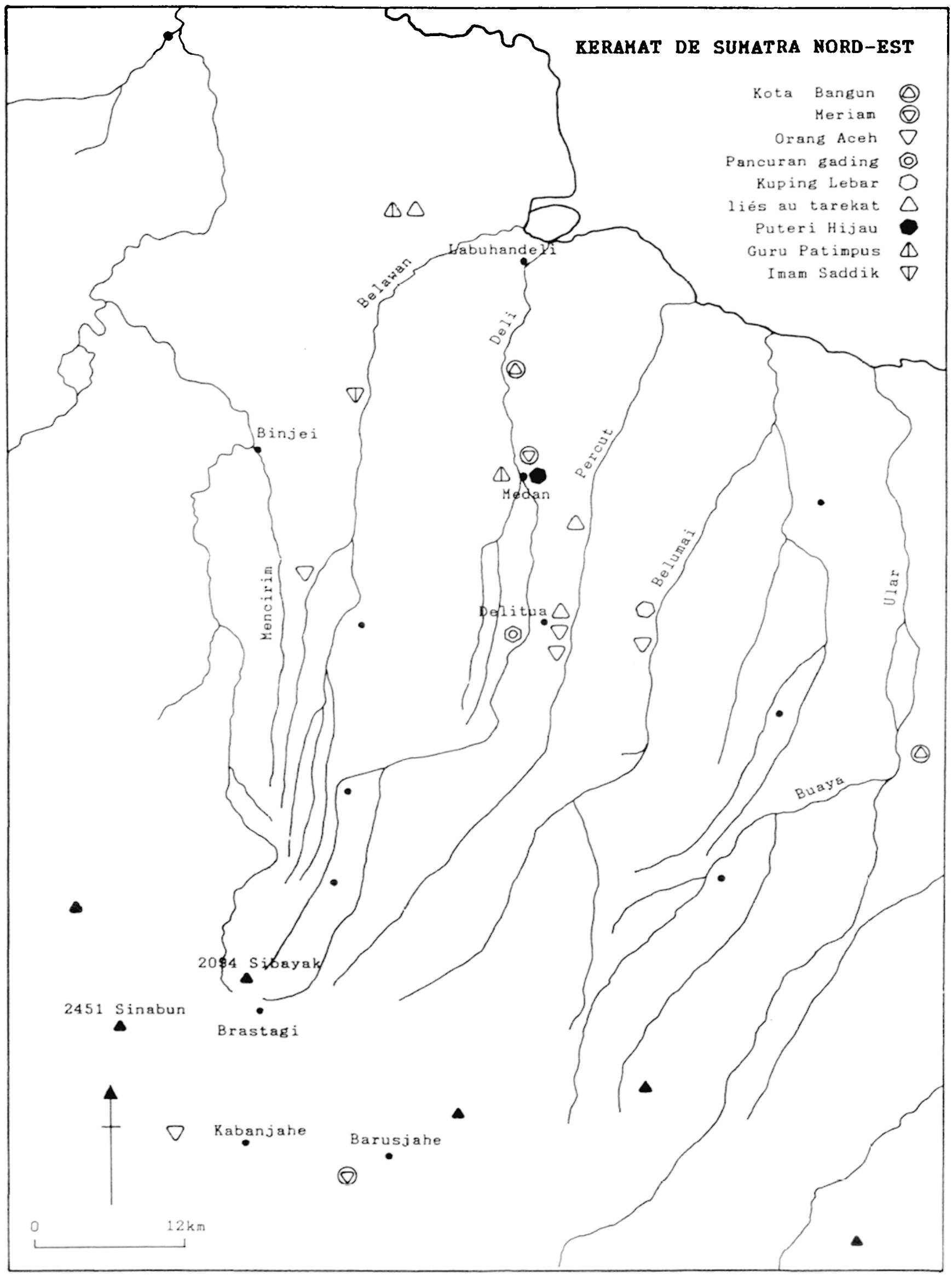


toire de ce qui deviendra le sultanat de Deli, d'un certain Mohamed Dalek, alias Gocah Pahlawan, originaire d'Inde, et présenté comme le grand artisan des conquêtes territoriales d'Aceh sous le règne d'Iskandar Muda. Le récit le montre débarquant sur la côte, près de l'actuelle Medan, puis s'installant après un certain temps en amont de Deli Tua (60).

A quelque trois kilomètres à l'est de Deli Tua, sur un promontoire au-dessus de la rivière Percut, se trouve le keramat «Nenek Moyang Sultan Deli» (la tombe des ancêtres des sultans de Deli) qui pour certains est la tombe de Gocah Pahlawan, pour d'autres la tombe d'un nommé Panglima Hitam, venu du Cachemire. Près de là, un keramat appelé «Kuburan Keling», matérialisé par une tombe d'environ trois mètres de long, faite d'un amoncellement de galets, est censé abriter le corps d'un héros acihais originaire du «Pakistan». La présence de ces lieux sacrés ne suffit toutefois pas à prouver l'existence de Mohammed Dalek.

La Hikayat Deli précise que le fils de Gocah Pahlawan est nommé «général en chef» (Panglima) par Aceh (61). Une source hollandaise confirme qu'un des titres des dirigeants de Deli au XVIIe siècle est Panglima, titre utilisé généralement à l'époque en référence à des généraux acihais ayant attaqué Malaka (62). Or, nous savons qu'en août 1631 quarante embarcations de la marine d'Aceh ont quitté la rivière Deli pour rejoindre le roi de Johor afin d'attaquer ensemble Malaka (63). C'est à cette époque que l'espace acihais prend sa plus grande extension côtière, contrôlant non seulement la côte ouest de Sumatra jusqu'à Padang et la côte est jusqu'à Deli, mais également plusieurs territoires sur la côte ouest de la péninsule malaise, en particulier Kedah et Perak.

Au siècle suivant la situation de la côte nord-est est instable. La région est l'objet d'un conflit de souveraineté entre Aceh d'une part et Siak d'autre part. En 1781, Radermacher note que Deli est dépendant d'Aceh (64), ce que Marsden confirme quelques années plus tard en signalant que la limite du royaume d'Aceh sur la côte est se situe non loin de la rivière Batubara (au sud de Serdang) (65).

L'alternance des soumissions se poursuit au XIXe siècle jusqu'à l'arrivée des Néerlandais en 1862. En 1814, Siak accorde le titre de sultan au souverain de Deli. Huit années plus tard, Anderson, lors de sa visite sur la côte est, affirme qu'Aceh a cessé d'exercer toute autorité sur Langkat et Deli (66). Mais en 1854, le sultan de Deli, Osman Perkasa Alam, est soumis par Aceh qui envoie contre lui une flotte de plus de deux cents embarcations sous la conduite de Pangeran Husin. Il semble alors être contraint de porter le titre de «Wakil sultan Aceh» (représentant du sultan d'Aceh), recevant d'Aceh le cap sembilan (nonuple sceau), le bawar (une arme blanche), et l'autorité sur la région allant de Tamiang à Asahan (67). Les autres dirigeants de la côte est auraient alors été dans l'obligation de passer par Deli pour toute requête auprès du sultan d'Aceh (68).

Peu après la visite de Netscher à Deli en 1862, les Acihais réapparaissent sur la côte nord-est avec une petite flotte de treize embarcations. Cette expédition, dont le but est de réaffirmer l'autorité d'Aceh sur la région, visite Serdang et Asahan mais ne peut entrer ni à Deli, ni à Langkat en raison de la pré- 
sence de bateaux hollandais (69). Parmi les indices de cette influence politique acihaise dans l'arrière-pays immédiat de Deli, figurent les titres d'origine acihaise portés par les dirigeants des différentes principautés (urung): celui de datuk à Sunggal, Hamperan Perak et Sukapiring (70), et celui de kejuruan à Senembah et Percut (71). L'apparition de ces titres dans la région n'est pas datable avec précision. On sait toutefois que celui de tengku, également d'origine acihaise (72), utilisé pour désigner les descendants des sultans de la côte nord-est, n'existe pas dans la Sejarah Melayu dont la rédaction remonte au début du XVIIe siècle (73). L'adoption de ces titres ne semblerait pas par conséquent antérieure au règne d'Iskandar Muda (74).

A un moment donné, les différentes principautés de Deli se fédèrent dans une institution portant le nom de «Datuk Empat Suku» ou «Datuk Berempat». Une organisation similaire se retrouve sur la côte ouest d'Aceh avec les «Tuha Peuet», chez les Gayo avec les «Pojo Si Opat», chez les Alas avec les «Raja Si Empat», au nord du lac Toba avec les «Raja Berempat», à Simalungun avec les «Raja Maropat», au sud du lac Toba à Silindung avec les «Raja Na Opat» (75). Les sources divergent sur l'époque de la mise en place de ce système sur la côte nord-est. La Hikayat Deli fait remonter sa création à l'époque de Sultan Amaluddin Panglima Mangedar Alam, au début du XIXe siècle (76). Dans la généalogie des dirigeants de Deli, ce personnage est effectivement le premier à porter le titre de sultan. Les traditions migratoires du nord du lac Toba, les généalogies et la Riwayat Hamperan Perak, qui raconte l'histoire de la lignée des dirigeants de la principauté de Hamperan Perak à Deli, affirment quant à elles que les ancêtres de ces datuk et kejuruan seraient issus de l'arrière-pays. Convertis à l'islam, certains membres de la lignée auraient eu à un moment ou à un autre des contacts avec Aceh.

Ainsi Guru Patimpus, pour les uns, petit-fils d'un des Si Singamangaradja, les «rois-prêtres» installés sur la rive sud du lac Toba, pour les autres, originaire du plateau au nord du lac Toba, se serait converti à l'islam en arrivant sur la côte est, puis aurait envoyé ses deux derniers fils étudier à Aceh. Ils en seraient revenus avec de nouveaux noms donnés par le sultan d'Aceh ainsi que le grade de "général en chef». C'est le descendant de l'un d'eux, à la cinquième génération, qui serait devenu le premier datuk de la principauté de Hamperan Perak. Un autre fils de Guru Patimpus se serait converti à l'islam pour diriger la principauté de Sukapiring. Son descendant à la troisième génération aurait pris le titre de datuk.

Quant à l'ancêtre du premier datuk de la principauté de Sunggal, il serait originaire du plateau au nord du lac Toba. Son arrière-petit-fils aurait été le premier à porter le titre de datuk. Selon la Hikayat Deli, la sœur de ce dernier aurait épousé Gocah Pahlawan. En ce qui concerne la principauté de Senembah, nous avons vu que Si Emblang Pinggol est supposé être le père du premier kejuruan. Marié à une acihaise lors d'un séjour dans le sultanat, son fils aurait été lui-même, selon les traditions, nommé kejuruan par le sultan d'Aceh. D'autre part, le récit de migration du groupe patrilinéaire exogame (merga) appelé Barus nous affirme que Si Mbelang Cuping s'en va, une fois devenu riche sur la côte nord-est, chercher un titre à Aceh (77). Enfin, d'après 
le manuscrit contenant le récit de migration du groupe Kembaren, l'individu qui vient du nord du lac Toba pour s'installer sur la côte (près de l'actuelle Medan), détient une lettre royale «à neuf sceaux» (cap si siwah). La similitude est troublante avec le nonuple sceau probablement adopté à Aceh sous le règne d'Iskandar Muda ${ }^{(78)}$.

\section{Un arrière-pays difficile à contrôler}

Si les marques de l'influence d'Aceh sur le plateau situé au nord du lac Toba sont encore plus fragmentaires, elles permettent toutefois d'affirmer l'existence de relations politiques anciennes. Il nous semble intéressant de souligner d'abord qu'en acihais les termes «Haro» ou «Karu» désignent «l'état d'agitation et de trouble d'un territoire» (79). Or, on sait que le plateau situé au nord du lac Toba est traditionnellement identifié dans les cartes ethniques comme le «pays Karo Batak».

Dans la Riwayat Hamperan Perak, Guru Patimpus parle d'Aceh en ces termes: «Raja kitapun ada di sana» («notre roi est là-bas») (80). La Hikayat Acéh, rédigée à l'époque d'Iskandar Muda, livre une scène dans l'intérieur du pays où le sultan rencontre un «Batak» qui l'interpelle avec l'expression «tuan kita» (notre maître) (81). Junghuhn recueille également au sud du lac l'information selon laquelle le roi d'Aceh, au moment de l'apogée du royaume (début XVIIe siècle), a eu une influence politique sur les autorités de la région au nord du lac Toba, lesquelles lui payaient tribut (82). Il faut noter comme symbole de cette allégeance, qu'à la fin du XIXe siècle, des hauts personnages du plateau portent, à certaines occasions, une pièce de brocart acihais (83).

Avec l'installation, à partir de 1864 , des premiers fonctionnaires hollandais sur la côte nord-est, à Deli, dans la zone des plantations de tabac, on peut appréhender avec un peu plus de précision la teneur de ce que furent, probablement depuis une époque ancienne, les rapports entre le nord du lac Toba et Aceh. En effet, ceux-ci font notamment l'objet d'une surveillance toute particulière à partir du moment où éclate la guerre d'Aceh en 1873 . On voit alors le plateau s'animer; des groupes viennent du nord en quête de soutien, ou simplement pour aller prêter main-forte aux opposants au pouvoir colonial au sud du lac ou encore pour réaffirmer l'autorité d'Aceh sur la région. Dès le début de la guerre, le contrôleur de Deli demande aux personnages influents du nord du lac Toba de s'associer pour s'opposer à d'éventuelles intrusions de bandes originaires d'Alas ou d'Aceh (84). Pourtant, cette requête n'empêche pas la présence sur le plateau, dès le mois suivant, d'une «ambassade» acihaise composée de quinze individus et dirigée par un Arabe. Celui-ci envoie un message aux nobles de Deli leur demandant de l'argent et des fusils, le tout pouvant être livré chez les autorités de deux localités du plateau, Lingga et Kabanjahe (85). Il semble, qu'à cette occasion, la délégation ait procédé à des «changements politiques», rehaussant le statut d'autorités alliées et destituant certains personnages jugés trop proches des Hollandais (86). Le séjour de ce groupe venu d'Aceh, qui va se prolonger plusieurs mois sur le plateau (87), se terminera mal puisque trois des plus importants représentants y seront assassinés dans une embuscade ${ }^{(88)}$. Dans le même temps, un petit-fils de l'ancien sultan de 
Deli, Sultan Mangedar Alam, se rend avec un petit groupe au sud d'Aceh, dans les pays Gayo et Alas, afin de soulever la population contre les Occidentaux installés à Deli (89).

Quelques années plus tard, Aceh est engagé dans un autre conflit à Sumatra-Nord, celui qui oppose Si Singamangaraja XII aux Hollandais. Il semble que dès la fin des années 1870, des Acihais traversent fréquemment le plateau au nord du lac Toba et l'île de Samosir pour rejoindre le théâtre des opérations au sud du lac ${ }^{(90)}$. Alors qu'il se trouve dans la région en 1883, Hagen apprend ainsi qu'un groupe de cinquante à soixante Acihais vient de passer par Lingga pour aller soutenir Si Singamangaraja (91). Au mois d'avril 1887, une troupc d'environ soixante Acihais est signalée dans l'île de Samosir. Elle vient exhorter les habitants à entrer en guerre contre les Hollandais. Inquiets des suites d'une probable défaite contre ces derniers, les autochtones refusent de s'associer aux Acihais et les chassent ${ }^{(92)}$. En octobre 1888, près d'une centaine d'Acihais armés, probablement appelés par l'une des autorités de Lingga, sont signalés sur le plateau. Accompagnés de deux déserteurs européens, leur but semble être de susciter un soulèvement général et l'invasion de Deli. Ils profitent de l'occasion pour infliger des amendes aux dirigeants hostiles à Aceh. Début 1889, un groupe de soixante-dix Acihais armés et trois Européens sont signalés dans les hautes terres de Deli. Ils seraient venus offrir leur aide à Si Singamangaradja XII pour lutter contre le Gouvernement (93). En mars 1889 , trois lettres adressées à des chefs acihais par l'un des dirigeants de Lingga sont saisies à Penang. Elles invitent les destinataires à venir se joindre aux chefs du plateau afin d'envahir Deli et de brûler les fermes où sèche le tabac (94).

A la même époque trois courriers envoyés de la rive nord du lac Toba par un Acihais, Tengku Mohammad Said, aux sultans de Deli et Serdang et au pangeran de Bedagai, sont interceptés par un espion du Gouvernement. Son texte, traduit ci-dessous, montre à l'évidence qu'Aceh considère encore à l'époque la région comme appartenant à sa sphère d'influence (95):

(Sceau de «Tongkoe Moeda Mohammad Said Atjeh») Le 6 du mois de jumadilawal 1306

«Ci-après, nous portons à votre connaissance que nous, en tant que délégué (wakil) du sultan d'Aceh, parcourons tous les territoires sous la domination du sultan et où les hulubalang honorent encore le sultan. Traversant les pays batak, nous constatons que Si Lima Kuta et Purba s'opposent au sultan. Les raisons pour lesquelles nous menons la guerre tiennent dans les neuf points suivants. Premièrement, ils ne veulent pas recevoir nos missives. Deuxièmement, ils ne font pas confiance à nos délégués (wakil). Troisièmement, ils offensent la bannière du sultan d'Aceh qu'ils considèrent comme un torchon rouge. Quatrièmement, lorsque nous passons près de leurs villages, ils rassemblent leurs gens et se prennent pour le sultan d'Aceh. Cinquièmement, lorsque nos gens les ont rencontrés et sont revenus, ils ont hurlé, nous traitant comme si nous étions de petits enfants. Sixièmement, ils sont venus nous ont... (?). 
Septièmement, notre jeune frère a été blessé par une balle. Huitièmement, lorsqu'installés à Paribuan, nous nous sommes rendus dans leur kampong de Sicka Rotong, pour demander s'ils voulaient encore reconnaître l'autorité du sultan d'Aceh, ils sont alors venus devant nous en disant que nous n'avions rien à faire chez eux et qu'ils nous chasseraient avec des armes. Ils ont ensuite tiré sur nous. Neuvièmement, ils veulent tuer Raja Selo. Ils ont attaqué sa maison et tué Panglima (?), panglima du sultan d'Aceh. Ils l'ont décapité, suspendu et offensé, offensant par là-même le sultan d'Aceh.

«Si après leurs fautes, ils ne veulent pas se racheter suivant l'adat du sultan d'Aceh, nous les ferons quitter Si Lima Kuta et Purba afin qu'ils ne restent plus dans le territoire du sultan d'Aceh. En ce qui concerne les princes hulubalang du sultan d'Aceh, s'ils célèbrent encore le sultan, le préjudice causé au sultan les concernent également. Que les princes nous aident avec le matériel de guerre nécessaire, armes, plomb, (?), et argent. En ce qui concerne le nombre de (?), il est suffisant. Les Acihais sont maintenant cinq cents. Dans environ vingt jours, il en viendra mille de plus que nous avons déjà mandés. De plus, si vous célébrez encore activement le sultan, ne soutenez pas Si Lima Kuta et Purba, car ils sont rebelles.

«Par ailleurs, ne soyez pas (?), nous n'attaquerons pas un royaume qui n'est pas rebelle au sultan d'Aceh. En ce qui concerne Raja Daloh, qui au nom des princes hulubalang du sultan d'Aceh, est venu nous voir pour réparer leurs fautes, (?), la chose est possible, s'ils veulent suivre l'adat du sultan d'Aceh.

«Nous attendons dix jours. Passé ce délai, nous attaquerons Si Lima Kuta. C'est ce que nous avons à dire.»

Vers la fin du XIXe siècle, les populations locales semblent particulièrement redouter ces incursions venues du nord (96). D'une part le plateau semble vivre dans l'angoisse des exactions, d'autre part le piémont craint qu'en cas de défaite des Hollandais, les Acihais ne viennent assassiner les populations en raison de leur compromission avec le pouvoir colonial (97). Il faut souligner que dans cette région, au moins à cette époque, la croyance selon laquelle les Acihais sont invulnérables est largement répandue ${ }^{(98)}$. Ces incursions sur le plateau et dans le piémont se poursuivent au moins jusqu'en 1912 et semblent à cette époque principalement menées pour se procurer du riz et des armes à feu dont certaines viennent en passant par différentes mains, des plantations de la côte. Si l'ensemble du plateau paraît bien subir la présence acihaise, certains personnages profitent de la situation pour renforcer et étendre leur zone d'influence. Ainsi, il en est parmi eux qui utilisent des «mercenaires» venus du nord. C'est le cas du sibayak de Kabanjahe, Pa Mbelgah, qui en 1893 s'est entouré d'un groupe d'Alas pour prendre le contrôle d'une partie du plateau (99).

Il y a aussi des indices plus difficilement datables de relations entre Aceh et le plateau. C'est le cas, près de Lingga, d'une tombe acihaise considérée comme keramat par les autochtones (100). Si toutes les traditions locales s'accordent sur l'idée qu'elle concerne un haut personnage acihais, elles 
divergent sur la présence réelle d'un cadavre (101) et sur l'époque de l'événement. Certaines en font un envoyé du sultan d'Aceh (102). Venu s'opposer à des groupes immigrants dans la région à partir du XVIIe siècle, il aurait été victime de ces affrontements (103). D'autres nomment ce personnage Tengku Syekh, situant sa venue dans la seconde moitié du XIXe siècle. L'histoire la plus élaborée renvoie à un combat mythique entre les esprits du ciel dont l'un d'eux tombe finalement sur l'île de Penang. Ses descendants vont à Aceh et Tengku Syekh serait un rejeton de cette lignée. Il se rend sur le plateau avec six compatriotes dans l'intention d'étendre son autorité et pour ce faire, écume la région pendant vingt jours, cherchant à réunir des partisans. Son action, qui rencontre peu de succès, ne fait qu'éveiller l'animosité de la population locale qui rapidement souhaite mettre fin à ses agissements. Mais dans les affrontements, ils sont repoussés par une force magique qui semble émaner du personnage venu du nord. Plusieurs traditions s'accordent toutefois pour terminer l'épisode par son assassinat par des gens du lieu (104).

Il semblerait qu'Aceh ait également joué un rôle dans l'activation, sur le plateau situé au nord du lac Toba, d'une institution similaire à celle des «Datuk Empat Suku» de la côte voisine, celle des «Raja Berempat». Dans ce domaine encore, l'essentiel des sources se compose de traditions. Ceci ne permet pas d'affirmer avec certitude l'époque de la mise en place de cette organisation ni le rôle effectif d'Aceh que l'on sait divisé, depuis une certaine époque, en quatre «tribus» (105). Certains auteurs affirment pourtant que cette institution a été importée dans la région par le sultanat d'Aceh (106). D'autres en font au contraire le résultat d'un mélange de l'adat minangkabau et de l'adat de Johor (107).

Les traditions affirment qu'autrefois le «haut gouvernement des pays Karo» était aux mains de «délégués» du sultan d'Aceh, à savoir les sibayak de Lingga, Barusjahe, Suka et Sarinembah, qui chacun avait autorité sur quelques urung (108). Après avoir réussi l'épreuve imposée par le sultan ou son représentant (certains pensent au Tengku Syekh ${ }^{(109)}$ ) qui aurait consisté à chevaucher (menunggang) un buffle, ces quatre hauts personnages auraient reçu les symboles de pouvoir, en particulier des armes blanches (110). Il semble toutefois que cette institution n'ait pas en fait joué un rôle important, les fonctions revêtant uniquement un aspect honorifique. En effet, à l'arrivée des Néerlandais, les «Raja Berempat» ne sont pas plus puissants que les autres raja et il est rarement question d'une action commune de leur part (111). Il faut souligner, par ailleurs, que comme dans la zone côtière, certains dirigeants portent le titre de kejuruan (112).

D'autres indices sont pour le moins surprenants dans la mesure où les informations livrées par les autochtones remettent en cause l'idée d'une «invention culturelle» propre aux «Batak» et aux «Karo» en particulier. Ainsi, lorsque Hagen se trouve en 1883 à Nagaseribu, près de Tongging, il remarque sur la très vieille maison du raja, des sculptures de six pieds de haut nommées singa qui lui dit-on, «sont l'œuvre d'un artiste acihais» (113). Le nom d'Aceh est même associé à des traditions religieuses qui n'ont rien de musulmanes. C'est le cas à propos de la cérémonie de Pakualuh sur le mont Kalaban près de 
Siberaya, rituel durant lequel les cadavres des raja de la merga Sembiring, auparavant conservés dans un coffre, sont brûlés. Les cendres sont ensuite déposées dans de petits bateaux sur la rivière Wampu pour rejoindre la résidence des esprits. Cette coutume isolée parmi les populations de l'intérieur est présentée par certains comme une punition infligée à la merga concernée par le sultan d'Aceh (114).

Comme le «plateau Karo», Simalungun conserve aussi des traditions où Aceh est l'initiateur de la division de la région en quatre principautés. Là également les «Raja Maropat» (les raja de Siantar, Tanah Jawa, Purba et Pane) sont considérés comme les représentants d'Aceh ${ }^{(115)}$. Le processus se serait déroulé à partir de deux anciens royaumes, ceux de Nagur et de Si Tanggang. Les sources divergent dans le détail, affirmant les unes que le roi de Nagur, ne se sentant pas assez fort pour gouverner tout ce territoire, en appela au sultan d'Aceh pour mettre en place de nouvelles règles de gouvernement, les autres que la partition en quatre principautés aurait eu lieu avant l'intervention d'Aceh. Pour la seconde catégorie de sources, le rôle d'Aceh se serait borné à l'attribution à chacun d'un titre et de symboles du pouvoir. Selon des témoignages occidentaux, deux de ces raja étaient toujours en possession de ces attributs à l'époque coloniale (116). Le fait que, dans les années 1930, le dirigeant de la principauté de Purba ait détenu encore un vieux sceau reçu du sultan d'Aceh, nous semble une preuve irréfutable de l'intégration, à un moment donné, de cette région dans la sphère d'influence politique acihaise.

\section{Le rôle d'Aceh dans l'islamisation du nord-est de Sumatra}

Nous savons, grâce à Marco Polo, que l'islam était présent dans le nord de l'île en 1291 (117). Cette mention est confirmée par la découverte de tombes musulmanes anciennes dans la région de Pasai: la tombe du premier sultan de Samudra-Pasai, Malik al-Salih (1297), celle de son successeur Malik al-Zahir (1326), ainsi que d'autres stèles datant du XIVe siècle (118). L'islamisation de la côte nord-est semble plus tardive. Mais des sites tels que Kota Cina ont pu, dès le XIIIe siècle, recevoir des marchands musulmans profitant du déclin et de la disparition de Srivijaya.

Ma Huan, le musulman chinois qui accompagne l'eunuque Zheng $\mathrm{He}$ dans sa mission de 1413, signale que les gens de Ya-lu (Aru) sont tous musulmans (119). La Sejarah Melayu confirme que le roi d'Aru est déjà musulman lorsqu'il se rend sur l'île de Bintan (en face de l'actuel Singapour) afin d'épouser la fille de l'ancien sultan de Malaka, Sultan Mahmud Shah, en exil après la prise de sa capitale par les Portugais. Le couple serait reparti à Aru avec une suite de quatre-vingts jeunes nobles fournie par le sultan (120). L'existence d'un roi musulman à Aru est confirmée par Pires en 1520 (121) et Pinto en 1539 (122).

La plus ancienne tombe musulmane de la région est celle de Imam Sadiq ibn Abdullah à Deli. Retrouvée à Klambir Lima, sur la rive gauche de la rivière Belawan, à une quinzaine de kilomètres de l'actuelle Medan, la stèle, de style acihais, indique qu'il s'agit d'un savant éminent, mort le 23 du mois Syaban de 998 H (27/06/1590 E.C. $)^{(123)}$. Cette tombe est maintenant appelée 
keramat «Kajang Batu» et les autochtones font du personnage enterré là l'un des islamisateurs de l'arrière-pays.

A en croire la Hikayat Deli, lorsque Mohamed Dalek arrive sur la côte de Deli dans la première moitié du XVIIe siècle, le premier dirigeant indigène qu'il rencontre est déjà musulman et sa localité possède une mosquée avec un imam, un khatib et un kadi (124). Dans les traditions locales, ces islamisateurs sont généralement dépeints comme des personnages extraordinaires, dotés de pouv.oirs surnaturels. Ainsi l'Acihais censé avoir fondé Deli aurait eu à faire usage de toute sa science pour dominer les spécialistes locaux de la religion traditionnelle. Il y serait parvenu notamment en traversant une rivière de manière extraordinaire. Pour empêcher qu'après sa mort, sa tombe (keramat Nenek Moyang Sultan Deli) ne devienne l'objet d'un culte, son successeur (?) aurait ajouté une tombe factice, préservant ainsi l'orthodoxie islamique (125).

Quant au fameux Tengku Syekh, dont le keramat se trouve sur le plateau près de Lingga, une tradition raconte qu'il a répandu l'islam en faisant croire aux gens qu'en lui donnant une partie du produit de leur travail, ils allaient augmenter leurs ressources et leurs récoltes. C'est ainsi qu'il serait devenu keramat pour les gens de l'urung Telu Kuru (126).

Ces idées de musulmans doués de pouvoirs extraordinaires renvoient peutêtre au souvenir d'actions de chefs de confréries mystiques (tarekat) dont la présence est attestée à Aceh depuis le XVIe siècle. Nous possédons à cet égard le témoignage du grand poète et mystique Hamzah Fansuri, qui vécut surtout à Aceh à la fin du XVIe siècle et a dévoilé son appartenance à la Qadiriyah (127). Au siècle suivant, Aceh, devenu centre majeur d'enseignement islamique, abrite des membres des tarekat Qadiriyah (128) et Syattariyah, cette dernière introduite notamment par Abdul Rauf de Singkel (129). La tarekat Naqsybandiyah a, quant à elle, peut-être été introduite dès la première moitié du XVIIe siècle à Sumatra est (130). Une tradition locale attribue d'ailleurs l'initiative de son introduction à l'un des fils de Guru Patimpus. Il serait en effet revenu d'Aceh accompagné de Imam Sadiq ibn Abdullah (mort en 1590), considéré comme un chef de cette tarekat (131).

\section{Conclusion}

Dans l'état actuel de nos connaissances, il n'est pas encore possible de tirer des conclusions définitives sur l'histoire des relations entre Aceh et Sumatra nord-est. Les indices relevés posent en fait plus de questions qu'ils n'apportent de réponses. Peut-on, par exemple, faire le lien entre les impératifs d'Aceh, à savoir la régularité d'acheminement des produits forestiers, du riz et des esclaves et l'émergence des «Raja Berempat» sur le plateau situé au nord du lac Toba et des «Raja Maropat» à Simalungun?

Aceh disposant des ressources de l'arrière-pays de la côte ouest (notamment du camphre et du benjoin, grâce au contrôle des comptoirs de Barus et Singkel), l'émergence ou la «réactivation» de ces institutions pourrait alors s'expliquer comme un moyen pour le grand sultanat d'obtenir des approvisionnements réguliers en s'assurant la fidélité de quelques hauts personnages. Cette stratégie peut également avoir été conçue comme la première étape vers 
une islamisation de la région. A ce titre, la mission du fameux Tengku Syekh ne constitue probablement qu'un épisode.

Si tel a été le but d'Aceh, son échec aura été double. Les «Raja Berempat» ne vont pas se convertir et leur prestige semble n'avoir jamais atteint le niveau escompté. Quant aux «Raja Maropat», s'ils s'entourent de conseillers, secrétaires et commerçants musulmans à partir du début du XXe siècle -celui de Siantar se convertissant même en 1905 avant d'être emprisonné et banni- il ne semble pas qu'Aceh ait joué un rôle dans cette avancée de l'islam à Simalungun.

On ne saurait toutefois minimiser le rôle politique d'Aceh sur les riverains septentrionaux et orientaux du lac Toba, car sa sécurité dépendait également des relations amicales ou autoritaires que le sultanat pouvait entretenir avec eux. Les missions successives des Acihais, au moins jusqu'à la fin du XIXe siècle, sur le plateau au nord du lac Toba pour réaffirmer une autorité politicojuridique sur toute la région, la présence attestée d'un sceau d'Aceh chez le Raja de Purba, certaines survivances culturelles, constituent, à notre avis, des preuves suffisamment solides à cet égard.

La question du rôle politique d'Aceh dans l'émergence du royaume de Deli reste encore à élucider. Son fondateur est-il un Acihais mis en place par Aceh pour assurer l'approvisionnement en riz et la sécurité d'un emplacement stratégique? Ce dignitaire a-t-il eu par la suite des vélléités d'indépendance obligeant Aceh à intervenir militairement? A-t-il été un Acihais «dissident»? Ainsi la fédération dite «Datuk Empat Suku» qui nous apparaît, au moment où nous disposons de sources suffisamment précises, comme une association de groupes territoriaux non exogames, pourrait s'expliquer par une volonté d'élaborer des systèmes d'alliance obligeant les membres de ces suku à remettre tout ou partie de leur production à un partenaire bien défini, en l'occurrence au dirigeant de Deli. Au prestige d'une origine acihaise, ce dernier aurait alors ajouté celui d'avoir réussi à multiplier les alliances pour asseoir son autorité.A l'aspect économique de ces institutions fédératrices, peut-être faut-il ajouter un aspect politique dans la mesure où Aceh avait besoin de combattants pour attaquer Malaka.

L'initiateur du «Datuk Empat Suku» est-il également à l'origine des plantations de poivriers à Deli? S'agit-il d'un pionnier venu du nord de l'île, à l'image du Néerlandais Jacob Nienhuys qui en 1863 viendra de Java y développer avec succès la culture du tabac, ou s'agit-il d' un autochtone qui, après un séjour à Aceh, est revenu tenter l'aventure? Aucune tradition locale ne mentionne explicitement la présence à Deli de planteurs acihais. Elles tendent plutôt à montrer que des autochtones auraient joué, peut-être par l'intermédiaire d'Aceh, un rôle non négligeable dans ce développement. Rien n'interdit de penser que la côte nord-est approvisionne déjà Aceh à partir de la seconde moitié du XVIe siècle lorsque le grand sultanat prend le contrôle de la côte pour un temps, mais il est peu probable que l'expansion des plantations de poivriers à Deli, si elle a bien eu lieu au cours du XVIIIe siècle, résulte d'une volonté politique du sultanat d'Aceh. Ce dernier se débat en effet à l'époque dans de grandes difficultés intérieures. Nous y verrions plutôt le signe d'une 
impuissance à empêcher le développement d'une structure politique économiquement concurrentielle qui, jusqu'à l'arrivée du pouvoir colonial en 1862, semble jouer sur une double allégeance Aceh/Siak pour conserver une autonomie de fait.

Il convient toutefois d'être prudent en ce domaine. En effet, au milieu du XIXe siècle, Aceh montre qu'il est encore capable de réactions par l'envoi d'une expédition armée à Deli en 1854. On ne sait pas dans quelle mesure, à partir de cette date, il ne récupère pas une part importante des revenus perçus par le sultan de Deli. Rien n'interdit de penser également que les séjours à Aceh d'autochtones liés antérieurement ou postérieurement aux plantations, comme le suggèrent les traditions, ne soient le signe de missions conférées par le sultanat à ces individus pour développer la production de poivre et répandre l'islam sur la côte nord-est. Au contraire de l'arrière-pays où Aceh échoue dans ce qui semble avoir été une tentative de conversion des personnages influents, la côte, déjà touchée par l'islam depuis au moins le début du XVe siècle, se présente comme un terrain favorable où les chefs d'urung vont se convertir et devenir les vecteurs importants de l'islamisation dans l'immédiat arrière-pays.

Le «Traité de Sumatra», conclu en 1871 entre les Hollandais et les Anglais permet aux premiers d'agir en toute liberté sur l'île, y compris à Aceh. Il débouche deux années plus tard sur la guerre d'annexion qui s'achèvera seulement vers 1906. Ce conflit se traduit, au nord du lac Toba, par la multiplication d'expéditions militaires hollandaises et précipite l'installation de l'administration coloniale pour protéger les plantations occidentales de la Côte Est, entreprises dont la mise en place a été assurée grâce à des interventions militaires dans les sultanats côtiers à partir de 1862. Cette évolution porte un coup fatal à l'unité de l'espace régional constitué par Aceh et le nord-est de Sumatra.

\section{ABRÉVIATIONS}

BEFEO Bulletin de l'Ecole Française d'Extrême-Orient

BKI Bijdragen tot de Taal-, Land- en Volkenkunde van de Koninklijk Instituut

JMBRAS Journal of the Malayan Branch, Royal Asiatic Society

JSEAH Journal of South-East Asian History

MNZ

OV

Mededeelingen van wege het Nederlandsch Zendeling-Genootschap

Oudheidkundig Verslag uitgegeven door het Bataviaasch Genootschap van Kunsten en Wetenschappen

PEFEO Publications de l'Ecole Française d'Extrême-Orient

PM Petermann's Mittheilungen

TBG Tijdschrift voor indische Taal-, Land- en Volkenkunde Bataviaasch Genootschap van Kunsten en Wetenschappen

TNAG Tijdschrift van de Nederlandsch Aardrijkundig Genootschap

VBG Verhandelingen van de Bataviaasch Genootschap 


\section{NOTES}

1. Daniel Perret, De la forêt primaire aux plantations coloniales: les deux ruptures politiques et socio-économiques de la côte est de Sumatra-Nord, BEFEO, 1994 (Sous presse).

2. C. Snouck Hurgronje, The Achehnese (trad. O'Sullivan, A.W.S.), Leiden: Brill, 1906, vol. 1: 9 .

3. Denys Lombard, Le sultanat d'Aceh au temps d'Iskandar Muda (1607-1636), PEFEO 61, Paris, 1967: 77.

4. Connues sous le nom générique de damar.

5. Telles que le getah majang, le getah perca et le getah rambung.

6. John Anderson, Mission to the East Coast of Sumatra in 1823, Edinburgh: W. Blackwood/London: T. Cadell Strand, 1826: 262, 295.

7. Notamment à Bohorok dans l'arrière-pays de Langkat. C. De Haan, «Verslag van eene Reis in de Bataklanden", $V B G, 38(2), 1875: 37$.

8. A Langkat. (A. de Beaulieu, «Voyage aux Indes Orientales», in Thévenot (éd.), Collections de voyages, tome II, Paris, 1666: 99).

9. Pour résumer notre point de vue sur la localisation d'Aru, nous pensons que cette entité politique voit son établissement principal se déplacer de Tamiang à l'embouchure de la rivière Barumum pendant son existence, du XIIIe siècle au XVIIe siècle. Pour une discussion détaillée de cette hypothèse, cf. Perret, De la forêt primaire aux plantations coloniales: les deux ruptures politiques et socioéconomiques de la côte est de Sumatra-Nord.

10. A. Cortesâo, (éd.) et (trad.), The Suma Oriental of Tomé Pires, Londres: Hakluyt Society, Series II, vols $89 \& 90,1944: 148$.

11. Lombard, 1967: 89.

12. Daghregister, 1644-1645: 125.

13. Daghregister, 1644-1645: 92.

14. J.H. Neumann, «Bijdrage tot de Geschiedenis der Karo-Batakstammen», $B K I, 83$, 1927: 176.

15. B. Hagen, «Rapport über eine im Dezember 1883 unternommene wissenschaftliche Reise an den Toba-See», TBG, 31, 1886: 355.

16. Daghregister, 1682(2): 929.

17. Ainsi, un certain Jooris Vermeeren, de passage à Deli en mai 1644, rapporte qu'il s'agit d'un pays très fertile d'où il est possible d'obtenir annuellement 300 à 400 last (le last correspond à environ $2000 \mathrm{~kg}$ ) de riz. Daghregister, 1644-1645: 125.

18. Lombard, 1967: 60-61.

19. Pires observe à propos d'Aru: «Le pays d'Aru regorge de riz très blanc et de bonne qualité [...]». Il indique également que Bata produit du riz. Cortesâo (éd.), 1944: 146, 148.

20. Jorge M. Dos Santos Alves, «Une ville inquiète et un Sultan barricadé: Aceh vers 1588», Archipel, 39, 1990: 105.

21. J.F. von Brenner, Besuch bei den Kannibalen Sumatras: Erste Durchquerung der unabhängigen Batak-Lande, Würzburg: Woerl, 1894: note 3, p. 157.

22. J.C.J. Kempees, De tocht van Overste van Daalen door de Gajo-, Alas- en Bataklanden, 8 Februari tot $23 \mathrm{Juli}$ 1904, Amsterdam, 1905: 189; Politiek verslag/Mailrapport 1422/1907; Politiek verslag/Mailrapport 1559/1907; Politiek 
verslag/Mailrapport 1716/1907; Politiek verslag/Mailrapport 2060/1908; Politiek verslag/Mailrapport 115/1910; Politiek verslag/Mailrapport 434/1910; Politiek verslag/Mailrapport 732/1912. Un rapport de 1907 donne la composition d'une de ces bandes: quatre Alas, un Gajo, six Aceh, deux Karo et deux Toba.

23. Beaulieu parle en effet de la déportation vers Aceh de 22000 personnes originaires des royaumes de Johor, Deli, Pahang, Kedah et Perak. Beaulieu dans Thévenot, 1666: 114.

24. H.N. van der Tuuk, De pen in gal gedoopt. Brieven en documenten verzameld en toegelicht door $R$. Nieuwenhuis, Amsterdam, 1962: 49. Le Pustaka Kembaren mentionne un commerce entre le nord du lac Toba et Langkat, via la rivière Wampu. Neumann, 1927: 176.

25. La première mention est dans le Ying-yai Sheng-lan, qui décrit une expédition maritime chinoise de 1413. J.V.G. Mills (éd.) et (trad.), Ma Huan, Ying-yai Sheng-lan, The Overall Survey of the Ocean's Shore (1433), Cambridge: Hakluyt Soc. Extra series 42, Cambridge Univ. Press, 1970: 118.

26. Plus au sud sur la côte est, on sait qu'en 1823 le poivrier vient d'être essayé dans l'arrière-pays d'Asahan (Cf. Anderson, 1826: 128, 322). Sur la côte ouest, Singkel fournit déjà du poivre au début du XVIe siècle (Cf. Cortesâo, 1944: 163) alors que Natal commence à en produire seulement à partir de 1810 et en exporte 500000 livres en 1823. Cf. Christine Dobbin, Islamic Revivalism in a Changing Peasant Economy: Central Sumatra, 1784-1847, Copenhagen, 1983: 172.

27. Dos Santos Alves, 1990: 105.

28. E. Netscher, «Togtes in het gebied van Riouw en Onderhoorigheden», TBG, 14, 1864: 345 .

29. C.E. Wurtzburg, «A Brief account of the several countries surrounding Prince of Wale's Island with their production. Recd. from Captain Leight in 1789», JMBRAS, XVI, Part I, 1938: 123-126.

30. Said Amat deviendrait même sultan de Siak en 1795. Cf. J.J. Mendelaar, Memorie van Overgave Padang en Bedagai, 1930: 12-13.

31. Comm. voor het adatrecht, «Het maleische gebied. Rechtsverhoudingen bij de peppercultuur (1913)», Adatrechtbundels, X, 1915: 253. Cf. également W.H.M. Schadee, Geschiedenis van Sumatra's Oostkust, 1919, T.1: 104.

32. Anonyme, «Aanvullingsnota van toelichting betreffende het rijk van Langkat», $T B G$, LIII, $1911: 328$.

33. Tengku Luckman Sinar, Sari Sejarah Serdang, 1986, T.1: 145-146.

34. B. Hagen, «Eine Reise nach dem Tobah-See in Zentralsumatra», $P M, 1883 b$ : 42, 43.

35. Tengku Luckman Sinar, 1986, T.1: 147-150.

36. J.H. Neumann, «Bijdrage tot de Geschiedenis der Karo-Batakstammen», $B K I, 82$, 1926: 9-10.

37. Daghregister, 1644-1645: 47, 76, 125. Daghregister, 1661: 273.

38. Hamilton signale en effet qu'il n'y a pas d'endroits de la côte de Sumatra entre Bengkalis et Aceh où les relations avec les marchands étrangers soient autorisées. A. Hamilton, A new account of the East Indies, Edinburgh, 1727, T.2: 126.

39. Cf. Netscher, 1864: 343.

40. Netscher, 1864: 347.

41. Cf. notamment H.N. van der Tuuk, Bataksch-Nederduitsch Woordenboek, Amsterdam: F. Muller, 1861: 14, 17, 33, 45, 109, 110, 115, 153, 228, 311, 326, $455,460,467$.

42. M. Joustra, Karo-Bataksch woordenboek, Leiden: E.J. Brill, 1907a: 81, 150, 197; 
J.H. Neumann, Karo Batak-Nederlands woordenboek, Medan: Varekamp \& Co, $1951: 38,58,204,218,333$.

43. Joustra, 1907a: 12, 47, 58, 103, 106, 147; Neumann, 1951: 12, 37, 104, 106.

44. Hagen, 1886: 352.

45. Lombard, 1967: 109.

46. Lombard, 1967: 106. Aceh émet ses monnaies d'or au moins depuis le règne de sultan Salah ad-Din (1530-1537). Cf. Teuku Ibrahim Alfian, Mata uang emas kerajaan-kerajaan di Aceh. Banda Aceh: Seri Penerbitan Museum Negeri Aceh $\mathrm{n}^{\circ} 3,1979: 28$.

47. On aurait retrouvé notamment deux récipients contenant des pièces d'or. L'un d'eux découvert en 1902 sur une plantation de Deli Tua contenait des pièces datées du dernier quart du XVIIe siècle, qui seraient aujourd'hui conservées au musée de Singapour. Les inscriptions sur une autre monnaie du XVIIe siècle, retrouvée près de Medan, indiquent qu'elle provient de Tarumun, un territoire au sud d'Aceh sur la côte ouest. Cf. Dada Meuraxa, Sejarah Kebudayaan Suku-Suku di Sumatra Utara, Medan: Sasterawan, 1973: 83-84.

48. De Haan, 1875: 16. Le nom vernaculaire de cette rémunération est «amas». Ce terme est également utilisé dans l'évaluation des amendes et des bijoux d'argent appelés padung padung. Neumann, 1951: 15.

49. M. Joustra, «Het leven, de zeden en gewoonten der Bataks», MNZ, 46, 1902a: 399. T. Bangun, 1986: 129.

50. R.H. Kroesen \& W.D. Helderman, «Mededeelingen betreffende het landschap Panei en het Rajahgebied», BKI, LVI, 1904: 569.

51. F. Mendes Pinto, Pérégrination (trad. Viale, R.), Paris: La Différence, 1991 [1ère éd. française, chez Cotinet et Roger, 1645]: 110.

52. Mendes Pinto, 1991: 101-102.

53. Mendes Pinto, 1991: 113.

54. Généalogie d'Iskandar Muda in: Lombard, 1967. Il aurait pris le titre de Sultan Ghori. Cf. J. Kathirithamby-Wells, «Achehnese control over West Sumatra up to the treaty of Painan, 1663», JSEAH, X(3), 1969: 457.

55. A.C. Milner \& T.L. Sinar \& E.E. McKinnon, «A note on Aru and Kota Cina», Indonesia, 26, 1978: 17; Teensma, «An unknown Portuguese text on Sumatra from 1582», BKI, 145(2-3), 1989: 309; Mendes Pinto, 1991: 113.

56. Lombard, 1967: 91-92.

57. W. Middendorp, «The administration of the outer provinces of the Netherlands Indies», in: B. Schrieke (éd.), The Effect of Western Influence on Native Civilisations in the Malay Archipelago, Batavia: Royal Batavia Society of Arts and Sciences, 1929: 158. Pour certains la légende se rapporte aux conflits de 1539 ou 1564.

58. Une autre version indique que l'armée d'Aceh ne peut prendre le fort de Deli Tua car il est entouré de bambous épineux. Les troupes d'Aceh répandent alors des dirham à travers les bosquets de bambous et se cachent. La population de Deli Tua, voyant les pièces d'or dans les bosquets, coupe les bambous, si bien qu'Aceh peut attaquer de nouveau et capturer Putri Hijau. Cf. Dada Meuraxa, Keradjaan Melayu Purba, Medan: Kalidasa, 1971: 75; Brahma Putro, Karo dari Jaman ke Jaman, Medan: Yayasan Massa, 1981: 232. Des subterfuges identiques apparaissent dans d'autres récits de sièges militaires en Asie du Sud-Est. Cf. A. Migot, Les Khmers - Des origines d'Angkor au Cambodge d'aujourd'hui, Paris: Le livre contemporain, 1960: 227-228 et Khin Sok, Les chroniques royales du Cambodge (de Bana Yat jusqu'à la prise de Lanvaek), Thèse 3ème cycle, université Paris IV, 1975: 375, 378-382, 419, à propos de la prise de Lovêk, la capitale 
du Cambodge, par les Siamois en 1593-1594. Cf. également P.B. Lafont, Aperçus sur le Laos, Saïgon: Comité de l'Alliance Française du Laos, 1959: 28, à propos de la prise de Phay Nam (Vientiane) au Laos par Fa Ngum au milieu du XIVe siècle.

59. A. Rahman (éd.), Sja'ir Puteri Hidjau, Jakarta: Balai Pustaka, 1955 [1 ère éd. 1924]. A Deli Tua, au pied d'un benteng, se trouve le keramat Pancuran Gading que les autochtones pensent être le lieu où se baignait la Putri Hijau. Il y a d'autre part un keramat Putri Hijau à Medan.

60. Hikayat Deli: 180. Toutes les traditions locales ne donnent pas une origine aussi élevée à la lignée des sultans de Deli. Ainsi une version de Putri Hijau conte la disparition d'une première lignée dont la succession aurait été reprise par un esclave du dernier sultan de cette première lignée. M. Joustra, "Iets over Bataksche literatuur», MNZ, 47, 1903:162-165.

61. «Il reçoit d'Aceh le titre de panglima et gouverne comme un sultan». (Hikayat Deli: 192).

62. Daghregister, 1644; A.C. Milner, Kerajaan. Malay Political Culture on the Eve of Colonial Rule, Tucson: The University of Arizona Press, 1982: 80, note 32, p. 148.

63. Daghregister, 08/1631.

64. J.C.M. Radermacher, «Beschrijving van het eiland Sumatra...», VBG, 3, 1781: 6.

65. William Marsden, Histoire de Sumatra, Paris, 1788 [1ère éd. anglaise 1783]. T.2: 218.

66. John Anderson, Acheen and the ports on the north and east coast of Sumatra, London, 1840: 22.

67. Schadee, 1919, T.1: 105.

68. S. Van der Plas, Memorie van Overgave Deli-Serdang, 1913: 59.

69. Tengku Luckman Sinar, «The Impact of Dutch Colonialism on the Malays Coastal States on the East Coast of Sumatra during the 19th century», in: Papers of the Dutch-Indonesian Historical Conference held at Noordwijkerhout, the Netherlands, 19 to 22 May 1976, Leiden/Jakarta, 1978: 182.

70. Tengku M. Lan Husny, Lintasan sejarah perabadan dan budaya penduduk Melayu Pesisir Deli Sumatra Timur (1612-1950), Jakarta: Dep. Pend. dan Keb., 1978: 201.

71. Van der Plas, Memorie van Overgave Deli-Serdang, 1910: 22. En acihais, keudjroeën est le titre de dirigeants de territoires dépendants du sultanat d'Aceh. (R. Dr. H. Djajadiningrat, Atjehsch-Nederlandsch Woordenboek, Batavia, 1934, T.1: 693).

72. Alors que sur la côte nord-est le titre de tengku est politique, il sert à désigner les ulama à Aceh. A Aceh, les descendants de raja portent le titre de teuku. Cf. Zahari, Peradaban Bangsa Melajoe, Medan: Persekoetoean Penerbitan Deliana, 1945: 43.

73. Il n'est pas certain que ce titre ait été utilisé par les souverains de Malaka. En revanche, à la chute de la dynastie, vers 1700 E.C., les fils du nouveau sultan de Johor adoptent ce titre à la place de celui de raja. R.J. Wilkinson, A MalayEnglish Dictionary (romanised), Londres: MacMillan \& Co, 1957 [1ère éd. 1932], T.1: 306; T.2: 1203.

74. Il faut souligner que dans la Hikayat Deli, lorque Mohamed Dalek arrive à Percut Sungai Lalang, un certain Tengku Kejuruan Hitam est à la tête d'un negeri qui compte également plusieurs datuk. Cf. Hikayat Deli: 167, 170, 172, 178. S'agit-il d'une transposition dans un passé "pré-acihais» d'une situation valable après l'instauration de titres originaires d'Aceh, d'Acihais qui auraient précédé Moha- 
med Dalek ou encore d'autochtones mis en place par le sultanat du nord? Il est bien difficile de répondre.

75. Husny, 1978: 92.

76. «En ce qui concerne les quatre $s u k u$ de Deli, le premier est le kejuruan de Senembah, le second est Serbanyaman, c'est-à-dire Sunggal qui est «Oeloen Djandji», le troisième est Dua Belas Kota (XII Kota), le quatrième Sukapiring et ainsi c'est à l'époque de Sultan Mangedar Alam qu'ont été nommés les datuk de ces $s u k u$. La nomination de ces quatre suku en fait les piliers du royaume». Cf. Hikayat Deli: 195.

77. Neumann, 1926: 10.

78. Cf. Lombard, 1967: 78-79.

79. Djajadiningrat, 1934, T.1: 570; 672.

80. B.S. Simanjuntak, Sejarah Batak, Balige: K. Sianapar, 1977: 218.

81. Lombard, 1967: 63.

82. F. Junghuhn, Die Battaländer auf Sumatra, Berlin: G. Reimer, 1847, T.1: 286.

83. Brenner, 1894: 273.

84. Mailrapport $687 / 1873$.

85. Mailrapport 113/1874; Mailrapport 152/1874; Mailrapport 179/1874.

86. Mailrapport 226/1874.

87. Hoos, Memorie van Overgave Sumatra Oostkust, 1886: 18.

88. Ass. res. Halewijn à Res. Sumatra Oostkust 30/04/1874. Geh. en Kab. E24/1874; Res. Locker de Bruin à GG 06/06/1874, Geh. en Kab. E24/1874.

89. Mailrapport $179 / 1874$.

90. Il semblerait qu'avant l'ouverture des hostilités en 1877, Si Singamangaradja XII se soit rendu à Aceh. (Hagen, 1886: 352).

91. Hagen, 1886: 352 .

92. Brenner, 1894: 129.

93. Mailrapport 107/1889.

94. Mailrapport 244/1889.

95. Mailrapport $96 / 1189$. Nous traduisons du néerlandais.

96. J.K. Wijngaarden, «De Zending onder de Karau-Bataks», MNZ, 37, 1893: 399. Cf. également C.J. Westenberg, "Verslag van eener reis naar de onafhankelijke Bataklanden ten noorden van het Tobameer», TNAG, XIV, 1897: 57, 64.

97. J. Claine, «Un an en Malaisie. Deli et les Bataks-Karo de Sumatra», Le Tour du Monde, LXIII , 1892: 372; J.K. Wijngaarden, «De zending onder de KarauBataks (Deli)», $M N Z, 38,1894 \mathrm{~b}: 78$.

98. Mailrapport 768/1888.

99. J.K. Wijngaarden, «Verslag omtrent de zending onder de Karau-Bataks over 1893», MNZ, 38, 1894a: 140-141.

100. La tombe est décrite comme suit par Von Brenner: «La 'tombe acihaise', située dans un petit jardin, est un monument bas en pierre dont les murets s'élèvent à peine à deux pieds au-dessus du sol et qui est recouvert par un toit «batak». Une toile blanche est étendue à l'intérieur. La tombe elle-même est caractérisée par deux stèles se faisant face dans l'axe longitudinal de l'ouvrage et qui symbolisent la tête et les pieds. Leur partie supérieure en forme de turban indique qu'il s'agit d'une véritable tombe musulmane». Cf. Brenner, 1894: 271. Le missionnaire Kruijt qui visite l'endroit en 1891 mentionne à proximité la présence d'une petite maison «batak» et d'un drapeau blanc sur une hampe. Il s'agit d'un balai, 
lieu de réunion des «chefs Batak». Cf. H.C. Kruijt, «Bezoekreis op het plateau van Deli (Karo-land)», MNZ, 35, $1891 \mathrm{~b}: 382$.

101. Ainsi le cadavre ne se trouverait pas à l'emplacement de la tombe, mais à Sukanalu, au pied du Mont Sinabung. Cf. Brenner, 1894: 271.

102. Brenner, 1894: note 3 p.157; Neumann, 1926: 25-26; ce même Neumann nomme l'endroit «beré-beren Tengku Atjih»(Neumann, 1951: 30); Brahma Putro, 1981: 226; Darwan Prinst \& Darwin Prinst, Sejarah dan Kebudayaan Karo, s.1.: Yrama, s.d. [1985]: 175 .

103. Neumann, 1926: 25-26.

104. Brenner, 1894: 271. Meuraxa, 1971: 74; Simanjuntak, 1977: 129; Darwan Prinst \& Darwin Prinst, 1985: 175.

105. Selon la tradition, cette division en kawom daterait du milieu du XVIe siècle. K.F.H. Van Langen, «De inrichting van het Atjehsche staatbestuur onder het Sultanaat", $B K I, 37,1888: 387$. D'après une chronique, une autre division du royaume d'Aceh, celle-là en trois districts ou sagoë avec respectivement 22,26 et 35 mukim, aurait eu lieu entre 1676 et 1678 . H. Millies, Recherches sur les Monnaies des indigènes de l'Archipel indien et de la Péninsule malaise, La Hay'e: $\mathrm{M}$. Nijhoff, 1871: 92 .

106. C.J. Westenberg, «Nota over de onafhankelijke Bataklanden», $T B G, \mathrm{XXXIV}$, 1891: 107; J.A. Kroesen, «Mededeelingen betreffende 6 Karo-landschappen», BKI, LXIII, 1909: 510; Neumann, 1926: 27, 34; Brahma Putro, 1981: 100. Joustra justifie sa position en affirmant que cette institution ne repose pas sur l'adat indigène. Cf. M. Joustra, Batakspiegel, Uitgave van het Bataksch Instituut $\mathrm{n}^{\circ} 3$, Leiden: S.C. van Doesburgh, 1910: 196.

107. Kroniek Oostkust van Sumatra-Instituut, 1916: 21.

108. Westenberg, 1897: 66.

109. Cf. Simanjuntak, 1977: 132.

110. E.J. Van den Berg, Memorie van Overgave Karolanden, 1934: 36-37; Meuraxa, 1971: 72-73; Brahma Putro, 1981: 209; Darwan Prinst \& Darwin Prinst, 1985: 18.

111. Westenberg, 1891: 108; Kroesen, 1909: 511; Joustra, 1910: 196; R. von HeineGeldern, «Le pays de P'i-k'ien, le roi au grand cou et le Singa Mangaradja», $B E F E O, 49(2), 1959: 376$.

112. Notamment à Betumbulan et Juhar. Westenberg, 1891: 110.

113. Hagen, 1886: 351 .

114. B. Hagen, «Beiträge zur Kenntnis der Battareligion», $T B G, 28,1883 \mathrm{a}: 524$.

115. P.A.L.E. van Dijk, «Rapport betreffende de Sibaloengoensche landschappen Tandjoeng Kassau, Tanah Djawa en Si Antar», TBG, XXXVII, 1894: 171-172; C.J. Westenberg, «Bataksche rijkes Dolok en Poerba», TNAG, XXII, 1905: 581; W.H.M. Schadee, De uitbreiding van ons gezag in de Bataklanden, Amsterdam, 1920: note 1, p. 18.

116. Kroesen \& Helderman, 1904: 558-559. J. De Ridder, Memorie van Overgave Serdang, 1933: 48.

117. Louis Hambis (éd.), Marco Polo. La Description du Monde, Paris: Klincksieck, 1955: 243.

118. Denys Lombard, Le carrefour javanais. Essai d'histoire globale, Paris: EHESS, 1990, II: 34.

119. J.V.G. Mills (éd.) et (trad.), 1970: 115.

120. C.C. Brown, Sejarah Melayu or "Malay Annals» a Translation of Raffles Ms 18 (in the Library of the R.A.S. London) with commentary, Kuala Lumpur: Oxford 
University Press, 1983 [1ère éd. 1970, 1ère publ. dans JMBRAS 25, 2-3, 1952]: 179-182.

121. Cortesâo (éd.) et (trad.), 1944: 147.

122. Mendes Pinto, 1991: 87.

123. J.P. Moquette, «De Graafsteen van Kloempang (Deli)», OV, II-III, 1922: 70-71.

124. Hikayat Deli: $167,174,177,178$.

125. Tengku Zikry, communication personnelle 1991.

126. Simanjuntak, 1977: 129.

127. Lombard, 1990, T.2: 119.

128. M. van Bruinessen, «The Origins and Developpement of the Naqshbandi Order in Indonesia», Der Islam, 67 (1), 1990: 157.

129. Lombard, 1990, T.2: 120.

130. Dobbin, 1983: 123.

131. H.A. Fuad Said, Syekh Abd. Wahab, Tuan Guru Babussalam, Medan: Pustaka Babussalam, 1988: 51. 